\title{
Sclerosing sialadenitis of the submandibular gland is rarely an immunoglobulin G4-related disease in the Finnish population
}

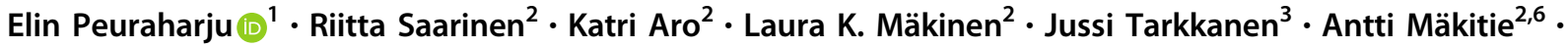 \\ Caj Haglund $^{4,5} \cdot$ Jaana Hagström ${ }^{3,4} \cdot$ Timo Atula $^{2}$
}

Received: 13 May 2019 / Revised: 1 October 2019 / Accepted: 1 October 2019 / Published online: 3 November 2019

(c) The Author(s), under exclusive licence to United States \& Canadian Academy of Pathology 2019

\begin{abstract}
Chronic sclerosing sialadenitis may represent one of many manifestations of an immunoglobulin G4-related disease. However, existing studies typically consist of small patient cohorts rarely conducted in Western populations. The clinical behavior of chronic sclerosing sialadenitis, including follow-up data, warrants further study. Thus, we aimed to determine whether chronic sclerosing sialadenitis always presents as IgG4-related disease or associates with autoimmune diseases and to determine which additional examinations patients may require. Between 2000 and 2017, 51 patients undergoing submandibular gland resection within the Helsinki University Hospital area were diagnosed with chronic sclerosing sialadenitis. We re-evaluated all specimens and performed immunostaining for IgG4. IgG and CD31 stainings were performed for IgG4-positive specimens. IgG4-related disease diagnosis was defined by the Boston consensus statement criteria. We revised clinical data, distributing a follow-up questionnaire to patients to register symptoms of IgG4-related disease or autoimmune disease during follow-up. The chronic sclerosing sialadenitis criteria were fulfilled in 34 patients, whereby 17 were diagnosed as non-sclerosing chronic sialadenitis. In 19 cases, a sialolith associated with a salivary gland lesion. In total, 12 of 51 cases were recognized as IgG4-positive, while two met the criteria for IgG4-related disease. These two cases belonged to the non-sclerosing chronic sialadenitis group, and both involved other organs. The histopathological features between chronic sclerosing sialadenitis and non-sclerosing chronic sialadenitis overlapped regarding the degree of fibrosis and inflammatory infiltrates. In the Finnish population, chronic sclerosing sialadenitis of the submandibular gland does not appear to present as IgG4-related disease. Non-sclerosing chronic sialadenitis can associate with IgG4-related disease. A histopathological distinction between chronic sclerosing sialadenitis and non-sclerosing chronic sialadenitis is not always unequivocal and the presence of a sialolith does not exclude IgG4-positivity. Therefore, immunostaining for IgG4 should be performed when dense plasma cell infiltration is present in either non-sclerosing chronic sialadenitis or chronic sclerosing sialadenitis.
\end{abstract}

\section{Introduction}

After the pancreas, the head and neck area, is the second most common site for immunoglobulin G4-related disease

These authors contributed equally: Jaana Hagström, Timo Atula

Elin Peuraharju

elin.peuraharju@helsinki.fi

1 Department of Oral and Maxillofacial Diseases, University of Helsinki, PO Box 41, FI-00014 Helsinki, Finland

2 Department of Otorhinolaryngology-Head and Neck Surgery, University of Helsinki and Helsinki University Hospital, PO Box 263, FI-00029 Helsinki, Finland

3 Department of Pathology, University of Helsinki, HUSLAB and
[1]. Previous definitions of chronic sclerosing sialadenitis, also known as Küttner's tumor, have recently evolved to include more specific pathophysiological features pointing towards IgG4-related disease. Thus, chronic sclerosing sialadenitis is now frequently regarded as an IgG4-related entity [2-4].

Helsinki University Hospital, PO Box 21, FI-00014 Helsinki, Finland

4 Research Programs Unit, Translational Cancer Medicine, University of Helsinki, PO Box 22, FI-00014 Helsinki, Finland

5 Department of Surgery, University of Helsinki and Helsinki University Hospital, PO Box 20, FI-00014 Helsinki, Finland

6 Research Program in Systems Oncology, Faculty of Medicine, University of Helsinki, Helsinki, Finland 
IgG4-related disease is considered an autoimmune disease and, many patients present with an elevated serum IgE level, infiltration of eosinophils into the tissue, and a history of allergies [5-7]. IgG4-related disease presents as a fibroinflammatory condition, clinically characterized by tumor-like lesions in the affected organ. Patients occasionally show elevated serum IgG4 levels [8], and histologically IgG4-related disease presents as dense lymphoplasmacytic infiltrates rich in IgG4-positive plasma cells and fibrosis arranged in a storiform pattern [9]. However, the pathological mechanisms as well as the etiology of IgG4-related disease remain unknown.

Chronic sclerosing sialadenitis typically presents as a palpable, firm submandibular gland and, thus, is often suspected as a malignant tumor before the final histopathological evaluation [10]. Prior to the introduction of a possible IgG4-related background, chronic sclerosing sialadenitis patients with a benign histology were offered neither further examinations nor clinical follow-up. However, since knowledge of IgG4-related disease has increased, the management of chronic sclerosing sialadenitis warrants re-evaluation. According to the existing literature, a significant portion of patients with IgG4related disease show multi-organ manifestations, possibly requiring further examination and follow-up [8, 10]. Whole-body scanning using computed tomography (CT) or positron emission tomography is recommended to evaluate the extent of disease [11].

Thus far, we have not encountered any recurrences or noted any relationship between chronic sclerosing sialadenitis and pathological manifestations in other organs in this patient population. Therefore, we hypothesized that not all chronic sclerosing sialadenitis cases truly represent a presentation of IgG4-related disease and, thus warrant further action. Moreover, globally chronic sclerosing sialadenitis might not behave in a similar manner, whereby a northern European patient series represents a valuable target population to further investigate.

In the present study, we retrospectively re-evaluated the histopathological samples and patient data from 51 consecutive patients who underwent submandibular gland resection and were diagnosed with chronic sclerosing sialadenitis. In addition, we performed IgG4 immunostaining, and analyzed patient outcomes to examine how many of our chronic sclerosing sialadenitis patients fulfilled the criteria for IgG4-related disease.

\section{Materials and methods}

We assessed the incidence of chronic sclerosing sialadenitis within the Helsinki University Hospital catchment district, which comprises 1.6 million inhabitants, and explored the proportion among all patients who underwent submandibular gland surgery between 1 January 2000 and 1 January 2017. The database for pathology at our institution (HUSLAB) was searched for chronic sclerosing sialadenitis, and we included all patients who underwent submandibular gland resection during the study period with a histopathologically confirmed diagnosis of chronic sclerosing sialadenitis. The local research ethics committee approved the study design, and we received institutional research permission for this study (HUS/967/2017).

\section{Histopathological re-evaluation}

Two experienced head and neck pathologists (JH and JT), who worked independently and were blinded to sample information, re-evaluated the original histopathological hematoxylin and eosin (H\&E)-stained submandibular gland specimens. The degree of fibrosis and lymphoplasmacytic infiltrates was evaluated, the presence of obliterative phlebitis and eosinophilia was assessed, and the original diagnosis of chronic sclerosing sialadenitis was re-evaluated. Thereafter, the samples were divided into two groups: chronic sclerosing sialadenitis and non-sclerosing chronic sialadenitis, based on degree of fibrosis and intensity of lymphoplasmacytic infiltrates [12]. In cases where the pathologists disagreed, specimens were evaluated in tandem and a diagnosis was assigned based on consensus.

\section{Immunohistochemistry}

The formalin-fixed submandibular gland samples were cut into $4-\mu \mathrm{m}$-thick sections. Prior to immunohistochemical staining, the specimens were prepared using deparaffinization in xylene, and rehydrated with graded ethanol and distilled water. For immunohistochemical analysis, the samples were stained for IgG4 using a mouse monoclonal anti-human IgG4 antibody (MCA2098G, BioRad, CA, USA). All cases with IgG4-positive plasma cell infiltrates with > 70 IgG4-positive plasma cells/high-power field (HPF; $40 \times$ ) were also stained for IgG using a rabbit polyclonal anti-human IgG antibody (A0423, Dako, Glostrup, Denmark). Antigen retrieval was performed in a PT module (LabVision UK Ltd, UK) in a Tris-EDTA buffer ( $\mathrm{pH}$ 8.5) for $20 \mathrm{~min}$ at $98{ }^{\circ} \mathrm{C}$. The sections were stained in an Autostainer 480 (LabVision) using the Dako REAL EnVision Detection System, Peroxidase/DAB +, rabbit/mouse (Dako, Glostrup, Denmark). Samples were treated with 0.3\% Dako REAL Peroxidase blocking for $5 \mathrm{~min}$. All slides were incubated with mouse monoclonal IgG4 antibody (1:100; CA, USA) for $1 \mathrm{~h}$ and cases with an elevated number of IgG4-positive plasma cells/HPF were incubated with the rabbit polyclonal IgG antibody (1:16,000 diluted in Dako REAL Antibody Diluent) for $1 \mathrm{~h}$, respectively, 
followed by a 30-min incubation with the peroxidaseconjugated Dako REAL EnVision/HRP, rabbit/mouse (ENV) reagent. Slides were visualized using the Dako REAL DAB + Chromogen for 10 min. Mayers Hematoxylin (Dako) was used for counterstaining.

The specimens showing IgG4-positive plasma cell infiltrates of $>70$ IgG4-positive plasma cells/HPF were additionally stained for CD31 to evaluate the obliterative phlebitis. Immunohistochemical staining for CD31 was performed using a mouse monoclonal (Clone JC70A) antihuman CD31 antibody (M0823, Dako, Glostrup, Denmark). The sections were stained in a BenchMark Ultra IHC/ISH slide-staining instrument (Roche) using the UltraVIEW DAB detection kit, mouse (Ventana Medical Systems, AZ, USA). The slides were incubated with a mouse monoclonal CD31 antibody (1:50; Dako, Glostrup, Denmark) for $1 \mathrm{~h}$.

\section{Evaluation of immunohistochemical stainings}

Plasma cells that stained positively for IgG4 were counted at three different HPFs in each case for hot spot areas. In cases with >70 IgG4-positive plasma cells/HPF, slides were additionally immunostained for IgG. In these specimens, the IgG-positive plasma cells were counted at three different HPFs as well, and the IgG4/IgG plasma cell ratio was calculated for these cases.

Based on the IgG4 staining, samples were further classified as either IgG4-positive or IgG4-negative and diagnosed as IgG4-related disease if the Boston consensus statement criteria were met [9]. The Boston consensus statement criteria for the diagnosis of IgG4-related disease in the salivary glands consist of: $>100 \mathrm{IgG} 4$-positive plasma cells/HPF, an IgG4/IgG plasma cell ratio $>40 \%$, and at least two out of three of histopathological features must be present. These features are a dense lymphoplasmacytic infiltrate with lymphoid follicle formation; fibrosis arranged, at least focally, in a storiform pattern; and obliterative phlebitis, sometimes absent in the salivary glands [9].

\section{Patient data}

We collected clinical patient data from the Helsinki University Hospital database, including patient age at the time of surgery, sex, type and duration of symptoms, imaging, pre-operative diagnosis, smoking, and significant comorbidities such as any autoimmune disease, asthma, and allergies. In addition, we gathered data on hospital visits regarding new symptoms or diagnoses after the chronic sclerosing sialadenitis diagnosis. Hospital records were reviewed in March 2019 (median time from diagnosis, 10.2 years; range, 0.7-16.0 years). In addition, all Finnish-speaking patients received a questionnaire along with a postage-paid return envelope. We queried any further symptoms related to the salivary glands or other conditions that developed following submandibular gland surgery (median time from diagnosis, 10.2 years; range, $3.0-14.8$ years).

\section{Results}

In total, 520 patients underwent submandibular gland surgery during the study period. The histopathological diagnosis was chronic sialadenitis (without sclerosis), sialolithiasis, or both in $320(62 \%)$ cases, and chronic sclerosing sialadenitis in $51(10 \%)$. All patients with a primary histopathological diagnosis of chronic sclerosing sialadenitis were included for further analysis.

The median age of patients was 60 years (range, 25-89 years), consisting of $26(51 \%)$ men and 25 women (49\%; Table 1). The main indications for surgery were: suspicion of a tumor $(n=23 ; 45 \%)$; recurrent infection or swelling

Table 1 Summary of clinical findings of patients $(n=51)$ initially diagnosed with chronic sclerosing sialadenitis and a comparison of clinical findings between patient groups after re-evaluation, that is, the group with confirmed chronic sclerosing sialadenitis vs the group with non-sclerosing chronic sialadenitis

\begin{tabular}{|c|c|c|c|}
\hline & $\begin{array}{l}\text { All patients } \\
n=51\end{array}$ & $\begin{array}{l}\text { Chronic } \\
\text { sclerosing } \\
\text { sialadenitis } \\
n=34\end{array}$ & $\begin{array}{l}\text { Non-sclerosing } \\
\text { chronic } \\
\text { sialadenitis } \\
n=17\end{array}$ \\
\hline Mean age (in years) & 57.6 & 59.0 & 54.6 \\
\hline Sex (male:female) & $26: 25$ & $18: 16$ & $8: 9$ \\
\hline $\begin{array}{l}\text { Mean duration of } \\
\text { symptoms (in years) }\end{array}$ & 2.6 & 3.2 & 2.3 \\
\hline $\begin{array}{l}\text { Intermittent } \\
\text { symptoms }\end{array}$ & 25 & 12 & 13 \\
\hline \multicolumn{4}{|l|}{ Smoking } \\
\hline Yes & 14 & 10 & 4 \\
\hline Former smoker & 4 & 2 & 2 \\
\hline Never smoker & 31 & 21 & 10 \\
\hline No data & 2 & 1 & 1 \\
\hline \multicolumn{4}{|l|}{ History of asthma } \\
\hline Yes & 6 & 3 & 3 \\
\hline No & 44 & 30 & 14 \\
\hline No data & 1 & 1 & 0 \\
\hline \multicolumn{4}{|l|}{ History of allergies } \\
\hline Yes & 15 & 7 & 8 \\
\hline No & 36 & 27 & 9 \\
\hline \multicolumn{4}{|c|}{ History of other autoimmune diseases } \\
\hline Yes & 5 & 4 & 1 \\
\hline No & 46 & 30 & 16 \\
\hline $\begin{array}{l}\text { Previously } \\
\text { diagnosed IgG4- } \\
\text { related disease }\end{array}$ & 2 & 0 & 2 \\
\hline
\end{tabular}


$(n=17 ; 33 \%)$; or sialolithiasis $(n=11 ; 22 \%)$. Salivary stones were removed in $19(37 \%)$ patients either before or during surgery or were observed during a pathological evaluation.

In 2 of the 51 patients the histopathological diagnosis of chronic sclerosing sialadenitis was stated to be IgG4related. In one of these two patients IgG4-related disease was suspected preoperatively due to a preceding IgG4related dacryoadenitis.

\section{Histopathological re-evaluation and immunohistochemistry}

Chronic sclerosing sialadenitis was confirmed by histological re-evaluation in $34(67 \%)$ cases, corresponding to the annual chronic sclerosing sialadenitis incidence of 1.25 / $1,000,000$ population. The remaining 17 cases did not meet the internationally reported diagnostic criteria for chronic sclerosing sialadenitis [12] (Fig. 1).

Plasma cells were identified in all 51 samples, while in 39 (76\%) samples the lymphoplasmacytic infiltrate could be described as dense. All samples showed some degree of fibrosis, and $26(51 \%)$ specimens featured fibrosis arranged in a storiform pattern. Obliterative phlebitis was not conspicuous upon H\&E staining in any of the specimens. Upon CD31 staining, obliteration of small venous channels by lymphoplasmacytic infiltrates appeared in 3 of 12 specimens. Table 2 summarizes the histopathological and immunohistochemical features for all 51 cases.

Among these 51 samples, 12 showed elevated levels of IgG4-positive plasma cells (range, 70-188 IgG4 plasma cells/ HPF) upon IgG4 immunostaining. Table 3 provides the immunohistochemical, histopathological, and clinical features of these 12 cases. Among the 12 IgG4-positive cases, six had an $\mathrm{IgG} 4 / \mathrm{IgG}$ plasma-cell ratio of $>40 \%$, only three of which exhibited $>100$ IgG4-positive plasma cells/HPF. Only two of three cases fulfilled the major histopathological features of IgG4-related disease described in the Boston consensus statement. Both showed storiform fibrosis and dense lymphoplasmacytic infiltrates, while one exhibited obliterative phlebitis as well (Fig. 2). Thus, 2 of 51 (4\%) samples, both belonging to the non-sclerosing chronic sialadenitis group,
Fig. 1 Among

520 submandibular glands removed from 2000 through 2017, 51 patients with an original diagnosis of chronic sclerosing sialadenitis were retrieved and divided into subgroups. This figure illustrates the division of these 51 patients into subgroups based on the histopathological re-evaluation and immunohistochemical staining

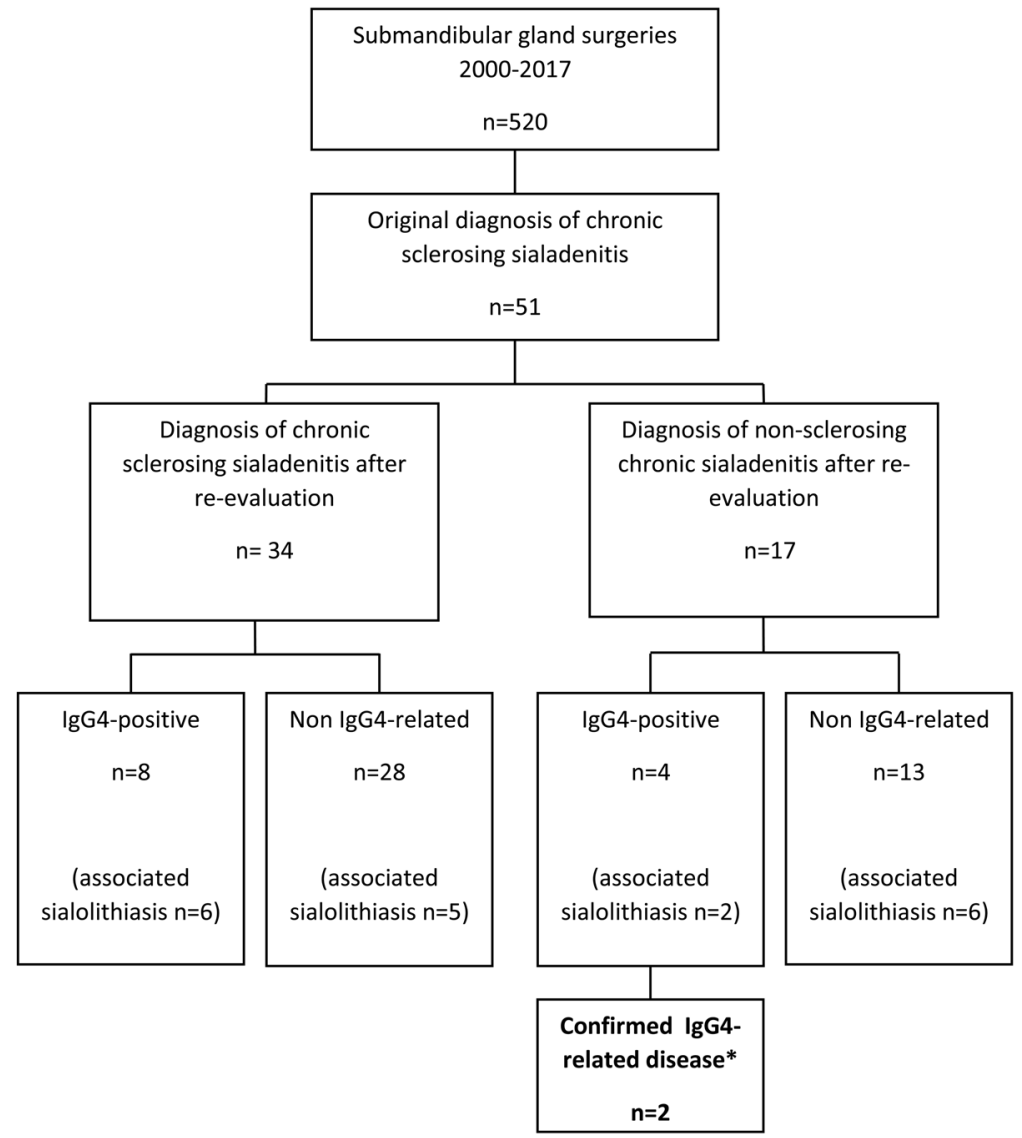

IgG4-positive, >70 IgG4-positive plasma cells /high power field; confirmed IgG4-RD, specimens fulfilling the Boston consensus statement criteria for IgG4-RD in the salivary gland.

* The two patients were diagnosed having IgG4-RD already before re-evaluation. 
Table 2 Based on the histopathological evaluation, all patients $(n=$ 51) were divided into two groups: chronic sclerosing sialadenitis or non-sclerosing chronic sialadenitis. This table summarizes the histopathological and immunohistological findings in chronic sclerosing sialadenitis and non-sclerosing chronic sialadenitis

\begin{tabular}{|c|c|c|c|c|}
\hline & \multicolumn{2}{|c|}{$\begin{array}{l}\text { Chronic } \\
\text { sclerosing } \\
\text { sialadenitis }\end{array}$} & \multicolumn{2}{|c|}{$\begin{array}{l}\text { Non- } \\
\text { sclerosing } \\
\text { chronic } \\
\text { sialadenitis }\end{array}$} \\
\hline & $n=34$ & $\%$ & $n=17$ & $\%$ \\
\hline \multicolumn{5}{|l|}{ H\&E-staining } \\
\hline Associated sialolithiasis & 11 & 32 & 8 & 47 \\
\hline Dense lymphoplasmacytic infiltrate & 29 & 85 & 11 & 65 \\
\hline Germinal centers with lymphoid follicles & 29 & 85 & 9 & 53 \\
\hline Fibrosis: storiform & 20 & 58 & 6 & 35 \\
\hline Fibrosis: dense & 28 & 82 & 15 & 88 \\
\hline Eosinophilic infiltrate & 1 & 3 & 1 & 6 \\
\hline \multicolumn{5}{|l|}{ IgG4-staining } \\
\hline$\geq 70$ IgG4 + plasma cells/HPF & 8 & 24 & 4 & 24 \\
\hline Additional IgG $^{\mathrm{a}}$ and $\mathrm{CD31}^{\mathrm{b}}$ staining & $\boldsymbol{n}=8$ & $\%$ & $\boldsymbol{n}=4$ & $\%$ \\
\hline IgG4/IgG-plasma cell ratio $\geq 40 \%$ & 3 & 38 & 3 & 75 \\
\hline Obliterative phlebitis & 1 & 13 & 2 & 50 \\
\hline
\end{tabular}

$H \& E$ hematoxylin and eosin, $H P F$ high-power field

${ }^{\mathrm{a}}$ IgG-staining was performed only in the 12 specimens showing IgG4positive plasma cell infiltrates $>70$ IgG4-positive plasma cells/HPF

${ }^{\mathrm{b}} \mathrm{CD} 31$-staining was performed only in the 12 specimens showing IgG4-positive plasma cell infiltrates > 70 IgG4-positive plasma cells/ HPF

were histologically highly suggestive of IgG4-related disease according to the Boston consensus statement [9]. These two patients were the only cases originally diagnosed as IgG4related disease before this study was initiated. Figure 2 illustrates the histopathological and immunohistochemical features of the confirmed IgG4-related disease -specimens.

In the remaining 39 cases, IgG4-positive plasma cells were either absent or present in small numbers, ranging from 0 to 62 IgG4-positive plasma cells/HPF, with a median of 5 IgG4-positive plasma cells/HPF.

\section{Clinical data}

Comorbidities across the entire patient series included asthma $(n=6)$, seropositive rheumatoid arthritis $(n=2)$, myasthenia gravis $(n=1)$, and psoriatic arthritis $(n=1)$. The patient with a preoperative suspicion of IgG4-related disease previously suffered from lacrimal gland swelling. In addition, 18 (35\%) patients were current or former smokers. The duration of symptoms varied from 1 month to 30 years (median, 7 months; mean, 2.6 years; no data for one patient). Symptoms appeared intermittently in 25 (49\%) patients. At the time of the chronic sclerosing sialadenitis -diagnosis, no patients had any symptoms related to other salivary glands.

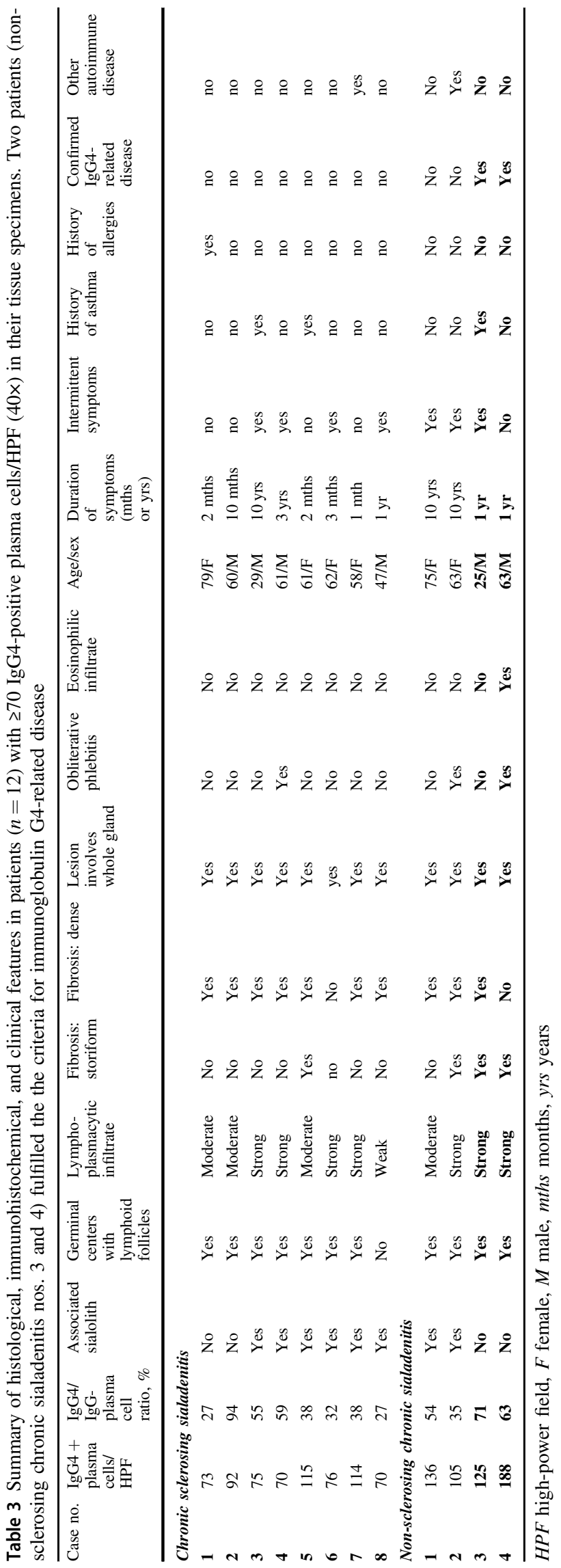



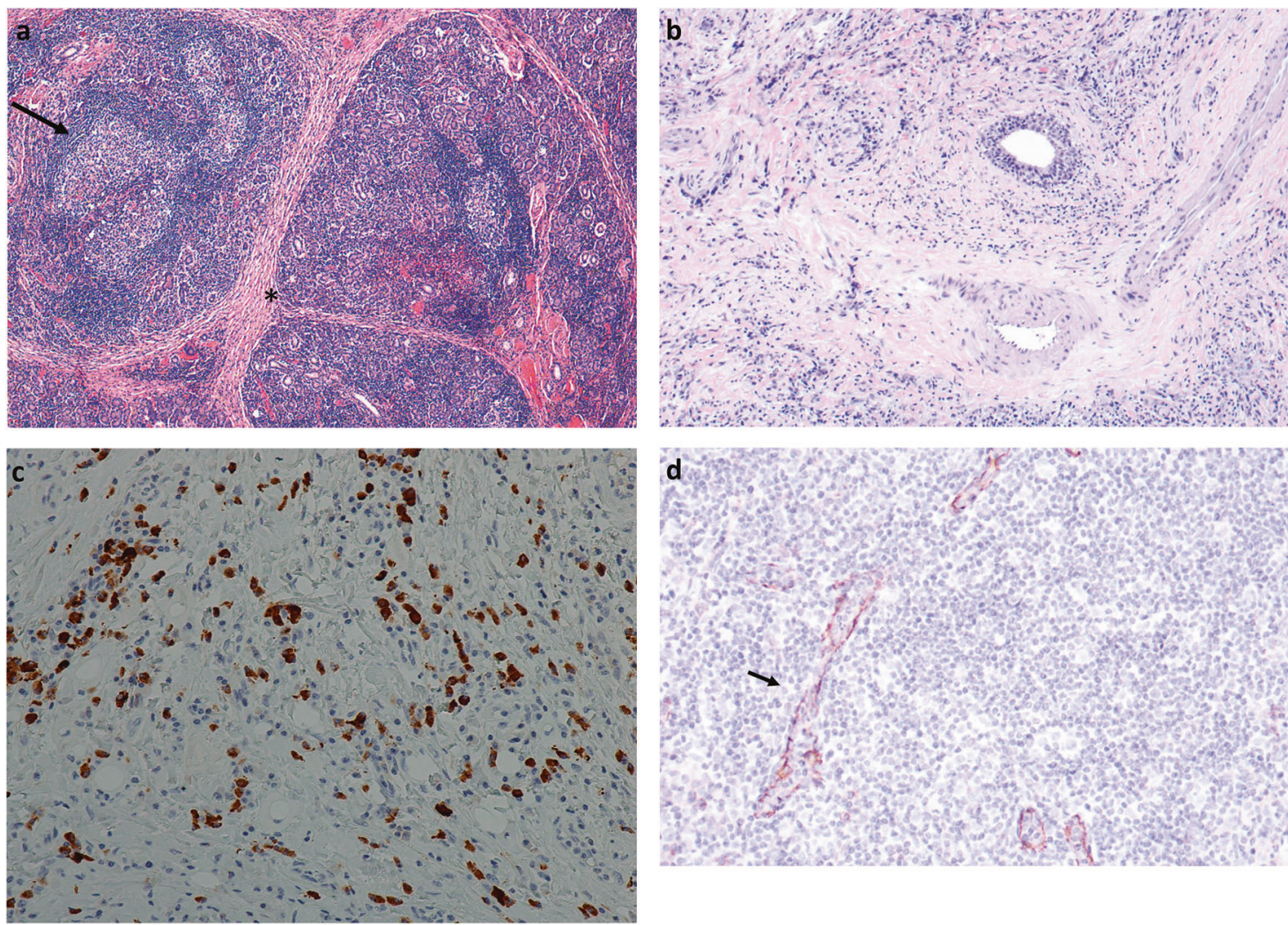

Fig. 2 Histological and immunohistochemical features of the tissue specimens from two patients with confirmed IgG4-related disease. a A dense lymphoplasmacytic infiltrate showing interlobular fibrosis (*) and irregular lymphoid follicles with germinal centers (arrow), hematoxylin, and eosin (H\&E), 4×. b Storiform fibrosis and an

Table 1 summarizes the clinical findings. Preoperative imaging included ultrasound examination in all but two patients, nine patients underwent further imaging with magnetic resonance imaging (MRI) and two with CT. For the two patients without a preoperative ultrasound, one had an MRI showing an intraglandular mass and the other was diagnosed via sialendoscopy as having a large intraglandular sialolith. No imaging data were available for one patient.

\section{Patient follow-up data}

According to hospital records, of the two patients with IgG4related disease, one had only a slightly elevated serum IgG4 level at the time of diagnosis and was treated for 7 months with oral prednisolone and has since remained symptomless. The other patient with confirmed IgG4-related disease had a clearly elevated serum IgG4 with persistent lung symptoms and was treated with oral prednisolone subsequently combined with azathioprine. This patient developed symptoms from the contralateral submandibular gland 2 years following the submandibular gland resection. In addition, two other patients developed rheumatoid arthritis later after the diagnosis of chronic sclerosing sialadenitis.

infiltrate of lymphocytes and plasma cells appear throughout the glandular tissue, H\&E, 10x. c Immunostaining for IgG4 showing abundant IgG4-positive plasma cells, 20×. d Immunostaining for CD31 showing inflammatory infiltrates obliterating the lumens of the small veins (arrow), 20x

A questionnaire about further salivary gland manifestations and other illnesses was mailed to 39 Finnish-speaking patients. Among the remaining patients, four died during follow-up, seven did not speak Finnish, and we could not find contact information for one patient.

Among the 39 patients who received the questionnaire, 29 (74\%) completed it. Only one patient (with a previously known IgG4-related disease) reported further symptoms related to IgG4-related disease (swelling of the contralateral submandibular gland). None of the other patients reported any further symptoms related to the salivary glands, autoimmune diseases or any diseases possibly associated with IgG4-related disease.

\section{Discussion}

In contrast to current understandings, in our Finnish cohort, chronic sclerosing sialadenitis was not associated with IgG4-related disease. Yet, a few patients with nonsclerosing chronic sialadenitis, without fulfilling the criteria for chronic sclerosing sialadenitis, exhibited IgG4related disease. Our study showed that the histopathological 
features between non-sclerosing chronic sialadenitis and chronic sclerosing sialadenitis overlapped. Furthermore, contrary to previous reports, our results showed that elevated numbers of IgG4-positive plasma cells can be present in specimens associated with sialolithiasis.

In recent years, the concept of chronic sclerosing sialadenitis as IgG4-related disease has become widely accepted [3]. Currently, chronic sclerosing sialadenitis is commonly regarded as a manifestation of IgG4-related disease in the submandibular gland $[2,5]$. Our results, however, disagree with these reports, since none of our patients with chronic sclerosing sialadenitis fulfilled the histopathological criteria for IgG4-related disease. Yet, IgG4 positivity was found in eight patients with chronic sclerosing sialadenitis, while the clinical data, including the follow-up questionnaire data, among these or any other patients in our series revealed no information that raised suspicion of true IgG4-related disease.

Patients with IgG4-related disease, in general, are over 50 years of age $[1,6,13]$. Furthermore, when the salivary glands are affected, the submandibular gland is most often involved $[1,5]$. The distribution between the sexes appears equal for IgG4-related disease in head and neck sites [1]. In our series, one of two patients with IgG4-related disease was notably younger, at the age of 25 years, than IgG4related disease -patients in general.

Most of the research and the larger $(n>15)$ patient series of IgG4-related disease in the head and neck area have been published from Asia [1, 6, 14]. Reports from Western countries primarily include case studies or small patient series [1, 4, 15, 16]. In a comprehensive review, Mullhollad et al. described a total of 484 patients with IgG4-related disease in the head and neck, among whom only $13.4 \%$ were reported from beyond Japan [1]. Therefore, it is possible that the characteristics of IgG4related disease vary globally depending upon patients' genetic backgrounds. Our results support this speculation. However, since data on IgG4-related disease is now rapidly accumulating, forming a recognized disease entity, clinicians should be aware of, suspect, diagnose, and treat IgG4-related disease. Furthermore, they should acknowledge the possibility, although rare, that the disease affects multiple organs.

Our series comprised data from a comprehensive surgical salivary gland material, including all submandibular glands removed due to chronic sclerosing sialadenitis over a 17-year period. During the re-evaluation, differentiating non-sclerosing chronic sialadenitis from chronic sclerosing sialadenitis proved challenging in some cases, since the histopathological features of these two entities appeared to overlap. In the histopathological re-evaluation, a total of 17 specimens, including two previously confirmed IgG4-related disease cases, showed histological features of non-sclerosing chronic sialadenitis rather than chronic sclerosing sialadenitis. This finding highlights the challenge of distinguishing nonspecific forms of chronic sialadenitis from chronic sclerosing sialadenitis, since they can share morphological features [17]. According to Harrison and Rodriguez [18], an increase in the lymphoplasmacytic infiltrates, fibrosis, and the formation of sialoliths in nonspecific chronic sialadenitis may represent progression of non-sclerosing chronic sialadenitis to a disorder reminiscent of chronic sclerosing sialadenitis. In their study of 129 patients with non-sclerosing chronic sialadenitis, elevated numbers of IgG4-positive plasma cells appeared in only three specimens. The authors speculated that the IgG4positive plasma-cell infiltrates could represent a transitory reaction, only present at a certain phase of the nonspecific chronic inflammation driving the pathogenesis of nonsclerosing chronic sialadenitis and the formation of sialoliths [18]. However, the role of IgG4 in the pathogenesis of chronic sclerosing sialadenitis and the nonspecific forms of chronic sialadenitis requires further study.

Based on our results, we can speculate that chronic sclerosing sialadenitis may actually represent a continuum of non-sclerosing chronic sialadenitis, in which the submandibular gland reacts to chronic inflammation with an increase in glandular fibrosis. The degree of fibrosis, especially when appearing in a storiform pattern, is a key histopathological feature differentiating non-sclerosing chronic sialadenitis from chronic sclerosing sialadenitis. Thus, an increase in fibrosis due to chronic inflammation results in difficulties distinguishing non-sclerosing chronic sialadenitis from chronic sclerosing sialadenitis histologically. Furthermore, fibrosis starts to predominate in the affected organ in prolonged IgG4-related disease, causing the histopathological features of IgG4-related disease to become less evident $[7,8]$. This phenomenon might hinder the diagnosis of IgG4-related disease during the late stages of disease, making it also more difficult to distinguish IgG4-related disease of the submandibular gland from non-IgG4-related forms of non-sclerosing chronic sialadenitis and chronic sclerosing sialadenitis. In our cohort, 17 specimens were diagnosed as non-sclerosing chronic sialadenitis following re-evaluation; among these, both IgG4-positivity and true IgG4-related disease were found. However, these specimens are likely to represent a select group of non-sclerosing chronic sialadenitis with histopathological features similar to those of chronic sclerosing sialadenitis. In addition to these samples, we had a total of 320 additional submandibular gland surgeries during the study period, from which the histopathology revealed a non-specific chronic sialadenitis, sialolithiasis or both. Thus, we have yet to determine how common and to what extent IgG4 expression is elevated in non-sclerosing chronic sialadenitis and in sialolithiasis. 
Only two specimens in this series met the Boston consensus statement criteria for IgG4-related disease with certainty. In addition, four additional specimens exhibited an $\mathrm{IgG} 4 / \mathrm{IgG}$ ratio greater than $40 \%$, but these specimens either lacked the major histopathological criteria or had a plasmacell count $<100 \mathrm{IgG} 4$-positive plasma cells/HPF. According to the Boston consensus, the $\operatorname{IgG} 4 / \mathrm{IgG}$ ratio represents a more powerful tool in the histopathological diagnosis of IgG4-related disease than the IgG4 plasma cell count. However, without other histopathological features, an IgG4/ IgG ratio $>40 \%$ alone is insufficient to definitively diagnose IgG4-related disease. Furthermore, IgG4-positive plasmacell infiltrates are not specific to IgG4-related disease and the presence of more specific histopathological features are necessary to differentiate IgG4-related disease from mimicking disorders [19]. In cases where only a portion of the histopathological criteria are met, elevated IgG4 levels in serum or evidence of other organ involvement can confirm the diagnosis [9]. None of our patients, except those with confirmed IgG4-related disease, exhibited additional symptoms suggestive of IgG4-related disease before or after submandibular gland surgery.

In our material, IgG4 positivity related to both chronic sclerosing sialadenitis and non-sclerosing chronic sialadenitis. In addition, according to our results, IgG4associated forms of chronic sclerosing sialadenitis and non-sclerosing chronic sialadenitis are histologically indistinguishable from non-IgG4-related forms of chronic sclerosing sialadenitis and non-sclerosing chronic sialadenitis, and, thus, IgG4-specific staining is required for diagnosis. Previous studies clearly distinguished sialolithiasis and IgG4-related chronic sclerosing sialadenitis, and these studies showed no IgG4-positive plasma-cell infiltrates in chronic sclerosing sialadenitis associated with sialolithiasis [20, 21]. However, our results disagree with those reports, since we found elevated numbers of IgG4 plasma cells in cases with sialolithiasis as well. Therefore, we recommend that all chronic sclerosing sialadenitis and non-sclerosing chronic sialadenitis specimens with dense plasma-cell infiltration undergo immunohistochemistry examined for IgG4. If IgG4 positivity appears, immunostaining for IgG should be performed, the $\operatorname{IgG} 4 / \mathrm{IgG}$ ratio should be calculated, and other histopathological features of IgG4-related disease should be thoroughly assessed.

Moreover, the serum IgG4-level measurement plays an essential role in patients with IgG4-related disease [6, 22]. Due to the retrospective nature of our study, we did not have data on these levels in our series, except for two patients with IgG4-related disease. However, based on our general understanding of non-sclerosing chronic sialadenitis regarding the more common etiological factors underlying the disease, and including also our series with
520 submandibular gland surgeries, we found no indication for measuring serum IgG4 during the diagnostic phase among patients with an enlarged single salivary gland without other suspected IgG4-related disease manifestations. Instead, we recommend determining the serum IgG4 level only when the patient has a clinical suspicion of IgG4-related disease, that is, clinical manifestations other than one in the salivary gland alone or when IgG4 positivity is detected from a tissue specimen.

A histopathological sample is needed to confirm a definitive IgG4-related disease diagnosis. Takano et al. [10] pointed out that both clinical and histopathological correlations form the basis for this. In our series, all patients underwent a submandibular gland resection. Some researchers suggest performing a salivary gland biopsy when IgG4-related disease is suspected [23, 24]. We argue that a large-needle biopsy might remain sufficient to yield adequate material for diagnosis in some cases. However, we emphasize that whenever any kind of neoplasm is possible, open biopsy is highly contraindicated. Open biopsy even from a benign neoplasm can compromise patient outcome e.g., by increasing the risk of tumor recurrence $[25,26]$. Furthermore, surgery of the submandibular gland always requires specific preoperative planning procedures [25].

If histopathology indicates IgG4-related disease and elevated numbers of IgG4-positive plasma cells are found, additional clinical and serological examinations to exclude other possible IgG4-related disease manifestations are warranted. A meticulous patient history and clinical examination, as well as serum IgG4 determination, represent the minimal requirements. Imaging with positron electron tomography combined with CT has proven useful in identifying multiorgan manifestations of IgG4-related disease [11]. However, according to our findings, this or other further imaging appears warranted only in the case of a true IgG4-related disease, but not when elevated IgG4 levels in the tissue specimen and local symptoms only are encountered.

Both of our patients with IgG4-related disease had another organ involved and one suffered from persistent lung symptoms. Both of these patients were treated with corticosteroids, the first-line treatment of IgG4 related disease. In the latter patient, corticosteroid treatment was later combined with azathioprine. The treatment of IgG4-related disease is presented in more detail elsewhere [27].

In our cohort, none of the patients with non-IgG4-related non-sclerosing chronic sialadenitis or chronic sclerosing sialadenitis developed symptoms indicative of IgG4-related disease during the follow-up period. Therefore, we argue that a patient given a pathological diagnosis of non-IgG4related non-sclerosing chronic sialadenitis or chronic sclerosing sialadenitis without other clinical or serological findings suspicious of systemic IgG4-related disease requires no further examinations or follow-up. 
In conclusion, chronic sclerosing sialadenitis in the Finnish population does not appear to represent IgG4Related disease, although in some cases with chronic sclerosing sialadenitis a high ratio of IgG4-positive plasma cells appeared in tissue specimens. The only patients with confirmed IgG4-related disease were histopathologically diagnosed as non-sclerosing chronic sialadenitis. Based on our findings, we conclude that global variation in the presentation of IgG4-related disease appears likely, at least in the submandibular gland. Furthermore, non-sclerosing chronic sialadenitis and chronic sclerosing sialadenitis remain difficult to distinguish histologically. Thus, it is possible that they represent a continuum progressing from the former to the latter over time. Moreover, the distinction between the IgG4-related forms of chronic sclerosing sialadenitis and non-sclerosing chronic sialadenitis from their non-IgG4-related counterparts require staining specific to $\mathrm{IgG} 4$.

\section{Compliance with ethical standards}

Conflict of interest The authors declare that they have no conflict of interest.

Publisher's note Springer Nature remains neutral with regard to jurisdictional claims in published maps and institutional affiliations.

\section{References}

1. Mulholland GB, Jeffery CC, Satija P, et al. Immunoglobulin G4related diseases in the head and neck: a systematic review. $\mathrm{J}$ Otolaryngol Head Neck Surg. 2015;44:24.

2. Geyer JT, Ferry JA, Harris NL, et al. Chronic sclerosing sialadenitis (Kuttner tumor) is an IgG4-associated disease. Am J Surg Pathol. 2010;34:202-10.

3. Geyer JT, Deshpande V. IgG4-associated sialadenitis. Curr Opin Rheumatol. 2011;23:95-101.

4. Putra J, Ornstein DL. Kuttner tumor: IgG4-related disease of the submandibular gland. Head Neck Pathol. 2016;10:530-32.

5. Stone JH. IgG4-related disease: nomenclature, clinical features, and treatment. Semin Diagn Pathol. 2012;29:177-90.

6. Li W, Chen Y, Sun ZP, et al. Clinicopathological characteristics of immunoglobulin G4-related sialadenitis. Arthritis Res Ther. 2015; 17:186.

7. Kamisawa T, Zen Y, Pillai S, et al. IgG4-related disease. Lancet. 2015;385:1460-71.

8. Stone JH, Zen Y, Deshpande V. IgG4-related disease. N Engl J Med. 2012;366:539-51.
9. Deshpande V, Zen Y, Chan JK, et al. Consensus statement on the pathology of IgG4-related disease. Mod Pathol. 2012;25:1181-92.

10. Takano K, Yamamoto M, Takahashi H, et al. Recent advances in knowledge regarding the head and neck manifestations of IgG4related disease. Auris Nasus Larynx. 2017;44:7-17.

11. Zhang J, Chen $\mathrm{H}, \mathrm{Ma} \mathrm{Y}$, et al. Characterizing IgG4-related disease with (1)(8)F-FDG PET/CT: a prospective cohort study. Eur J Nucl Med Mol Imaging. 2014;41:1624-34.

12. Hellquist H, Skalova A. Non-neoplastic Lesions. In: Hellquist H, Skalova A, editors. Histopathology of the salivary glands. 1st ed. Berlin, Heidelberg: Springer-Verlag, 2014. p. 23-65.

13. Frulloni L, Lunardi C. Serum IgG4 in autoimmune pancreatitis: a marker of disease severity and recurrence? Dig Liver Dis. 2011;43:674-5.

14. Himi T, Takano K, Yamamoto $M$, et al. A novel concept of Mikulicz's disease as IgG4-related disease. Auris Nasus Larynx. 2012;39:9-17.

15. Pettersson T. IgG4-related disease-a common denominator of many conditions previously regarded as being confined to a single organ. Duodecim. 2014;130:209-18.

16. Weitz-Tuoretmaa A, Laranne J, Paloneva T, et al. Chronic sclerosing sialadenitis-Kuttner tumor. Duodecim. 2013;129:2280-3.

17. Teymoortash A, Tiemann M, Schrader C, et al. Chronic sclerosing sialadenitis of the submandibular gland is not just a severe chronic sialadenitis. Am J Otolaryngol. 2003;24:278.

18. Harrison JD, Rodriguez-Justo M. IgG4-related sialadenitis is rare: histopathological investigation of 129 cases of chronic submandibular sialadenitis. Histopathology. 2013;63:96-102.

19. Yamamoto M, Takahashi H, Shinomura Y. Mechanisms and assessment of IgG4-related disease: lessons for the rheumatologist. Nat Rev Rheumatol. 2014;10:148-59.

20. Furukawa S, Moriyama M, Kawano S, et al. Clinical relevance of Kuttner tumour and IgG4-related dacryoadenitis and sialoadenitis. Oral Dis. 2015;21:257-62.

21. Kitagawa S, Zen Y, Harada K, et al. Abundant IgG4-positive plasma cell infiltration characterizes chronic sclerosing sialadenitis (Kuttner's tumor). Am J Surg Pathol. 2005;29:783-91.

22. Carruthers MN, Khosroshahi A, Augustin T, et al. The diagnostic utility of serum IgG4 concentrations in IgG4-related disease. Ann Rheum Dis. 2015;74:14-8.

23. Takano K, Nomura K, Abe A, et al. Clinicopathological analysis of salivary gland tissue from patients with IgG4-related disease. Acta Otolaryngol. 2016;136:717-21.

24. Takano K, Keira Y, Seki N, et al. Evaluation of submandibular versus labial salivary gland fibrosis in IgG4-related disease. Mod Rheumatol. 2014;24:1023-5.

25. Atula T, Panigrahi J, Tarkkanen J, et al. Preoperative evaluation and surgical planning of submandibular gland tumors. Head Neck. 2017;39:1071-7.

26. Aro K, Valle J, Tarkkanen J, et al. Repeatedly recurring pleomorphic adenoma: a therapeutic challenge. Acta Otorhinolaryngol Ital. 2019;39:156-61.

27. Khosroshahi A, Wallace ZS, Crowe JL, et al. International consensus guidance statement on the management and treatment of IgG4-related disease. Arthritis Rheumatol. 2015;67:1688-99. 